\title{
Updating our view of organelle genome nucleotide landscape
}

\section{David Roy Smith*}

Department of Botany, Canadian Institute for Advanced Research, University of British Columbia, Vancouver, British Columbia, Canada

Edited by:

Jun Yu, Beijing Institute of Genomics, China

Reviewed by:

Jim Leebens-Mack, University of

Georgia, USA

Hui Wang, Natural Environmental

Research Council, UK

${ }^{*}$ Correspondence:

David Roy Smith, Department of

Botany, Canadian Institute for

Advanced Research, University of

British Columbia, 3529-6270

University Blvd., Vancouver, British

Columbia, Canada V6T $1 Z 4$

e-mail:smithdr@dal.ca
Organelle genomes show remarkable variation in architecture and coding content, yet their nucleotide composition is relatively unvarying across the eukaryotic domain, with most having a high adenine and thymine (AT) content. Recent studies, however, have uncovered guanine and cytosine (GC)-rich mitochondrial and plastid genomes. These sequences come from a small but eclectic list of species, including certain green plants and animals. Here, I review GC-rich organelle DNAs and the insights they have provided into the evolution of nucleotide landscape. I emphasize that GC-biased mitochondrial and plastid DNAs are more widespread than once thought, sometimes occurring together in the same species, and suggest that the forces biasing their nucleotide content can differ both among and within lineages, and may be associated with specific genome architectural features and life history traits.

Keywords: Coccomyxa, GC content, mitochondrial DNA, plastid DNA, Polytomella, Selaginella, RNA editing

\section{INTRODUCTION}

Mitochondria and plastids are the products of ancient endosymbiotic events, involving a proteobacterium and a cyanobacterium, respectively (Lang et al., 1999; Palmer, 2003). Mitochondria arrived early and probably existed in the common ancestor of all eukaryotes (Gray et al., 1999). Plastids came later, first arising in the Archaeplastida (Plantae), and then being passed on laterally to diverse lineages through eukaryote-eukaryote endosymbioses (Archibald, 2009; Keeling, 2010). The genomes within contemporary mitochondria and plastids have been fashioned through coexistence and coevolution with their eukaryotic hosts, and in many instances have acquired bizarre and complex architectures (Palmer, 1985; Gray et al., 2004; Green, 2011).

Organelle DNAs boast an impressive, and often puzzling, array of sizes $(<10$ to $>1000 \mathrm{~kb})$, conformations (circular or linear), chromosome numbers (monomeric to highly fragmented), compactnesses ( $<10$ to $>90 \%$ non-coding DNA), and gene repertoires ( $<5$ to $>250$ genes). Moreover, many organelle genomes use a non-standard genetic code (Jukes and Osawa, 1993), and some employ complicated editing systems that alter the sequences of RNA transcripts (Covello and Gray, 1989; Simpson and Thiemann, 1995). One feature of organelle DNA that has proven to be relatively constant across lineages is its nucleotide composition. Almost all completely sequenced mitochondrial and plastid DNAs (mtDNAs and ptDNAs) have a high adenine and thymine (AT) content (Kusumi and Tachida, 2005; Min and Hickey, 2007). Various hypotheses have tried to explain this AT bias, but the topic remains poorly understood.

Recently, it was shown that guanine and cytosine (GC)-rich organelle DNAs do exist (Tsuji et al., 2007; Smith and Lee, 2008; Hecht et al., 2011). These genomes come from a small but diverse group of species, including various green plants and animals, and sometimes have linear conformations or undergo large amounts of post-transcriptional editing. Unraveling the mechanism responsible for their GC enrichment may help explain the near-ubiquity of AT-rich mitochondrial and plastid genomes throughout the eukaryotic domain, and could give insights into other aspects organelle genome architecture, such as the origins of RNA editing. The existence of GC-rich organelle genomes, however, is poorly chronicled in the scientific literature, even though these sequences could impact how we use organelle DNA for studying molecular evolution (Foster and Hickey, 1999).

This review showcases GC-biased organelle genomes and the species in which they are found. GC enrichment is discussed in context to mutation, recombination, population genetics, and genome architecture. It is emphasized that GC-rich mtDNAs and ptDNAs are more common than once thought - occasionally occurring together in the same species - and that the processes promoting GC enrichment can differ within and among lineages.

\section{THE NEAR-UBIOUITY OF AT-RICH ORGANELLE GENOMES}

The sequencing of large numbers of organelle genomes from diverse lineages has revealed an almost universal AT bias in $\mathrm{mtD}$ NAs and ptDNAs across the eukaryotic domain (Figure 1). Of the $\sim 2,500$ mitochondrial and plastid genomes that have been sequenced, as of January 1, 2012, most have an AT content above $50 \%$ (average $\approx 65 \%$; Figure 1 ). Convergent evolution to AT richness is found in other types of organelle-located DNAs, such as mitochondrial plasmids (Handa, 2008), nucleomorph genomes (Moore and Archibald, 2009), and the genomes of mitochondrial viruses (Wu et al., 2010). Moreover, the genomes of bacterial and eukaryotic endosymbionts and intracellular parasites tend to have higher AT compositions than those of their free-living close relatives (Pallen and Wren, 2007; Nowack et al., 2008; McCutcheon and Moran, 2010). 


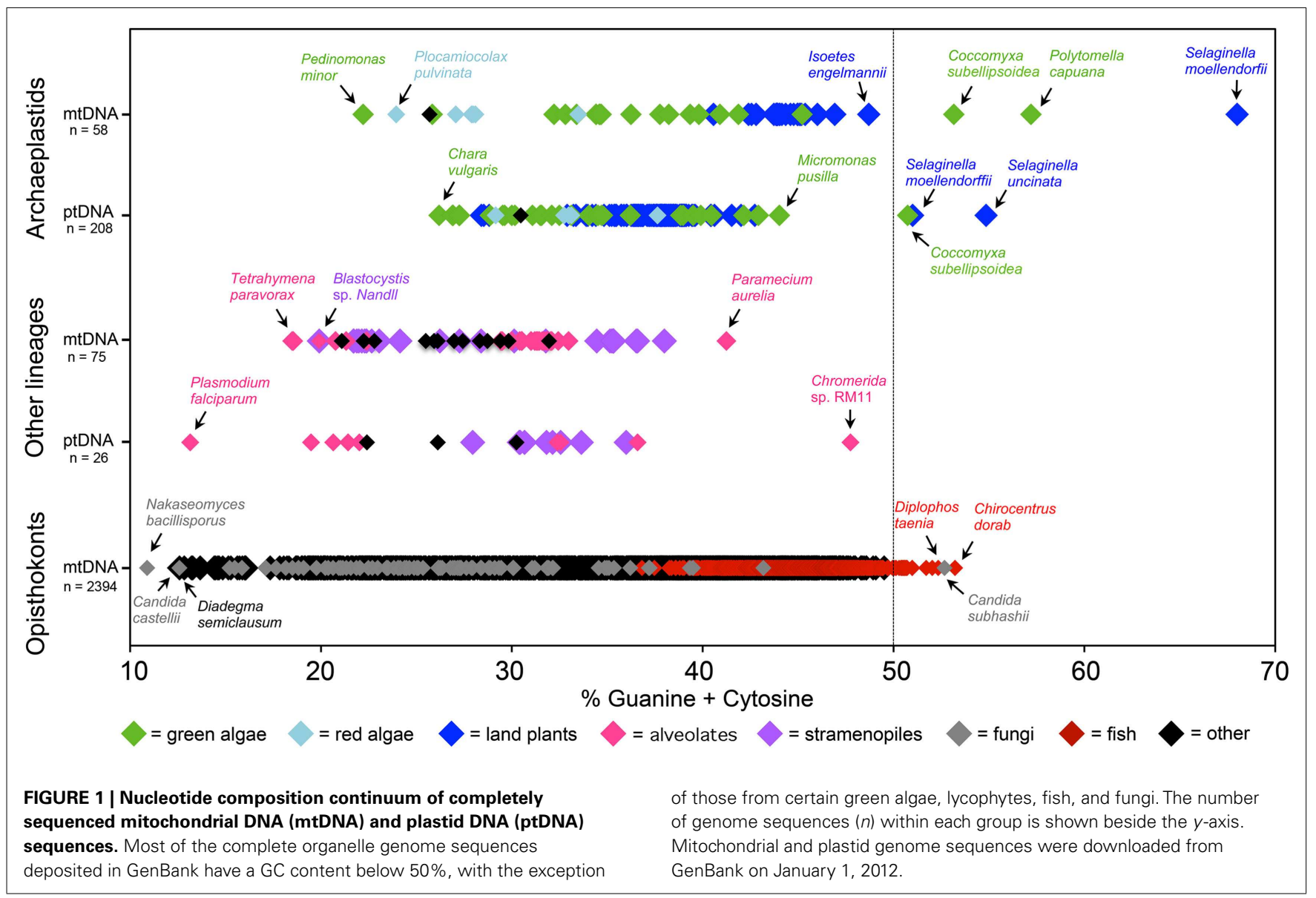

Many processes can influence nucleotide landscape, including mutation, recombination, random genetic drift, and selection (Lynch, 2007; Charlesworth and Charlesworth, 2010). The net effect of these processes ultimately determines the equilibrium nucleotide composition of a genome. The origins of AT richness within mtDNAs and ptDNAs are thought to reflect the endosymbiotic history of these genomes, their location within the cell, the unique population-genetic features that define organelles, and selection for metabolic and translational efficiency.

The massive shedding of genes that characterized early mtDNA and ptDNA evolution resulted, at least for some lineages, in the loss of key DNA repair proteins and, consequently, diminished nucleotide repair capacities within organelles (Kleine et al., 2009; Bendich, 2010, but see Liu and Demple, 2010). Organelle DNAs are typically uniparentally inherited, non-recombining, and can experience severe bottlenecks during transmission, which implies that they are inefficient at purging deleterious mutations from their populations (Muller, 1964; Rand, 2001; but see Piganeau et al., 2004). Organelle genomes undergo multiple rounds of replication per cell division (Birky, 2001), predisposing them to replication errors, and they are housed in energy-producing compartments where high concentrations of reactive oxygen species promote GC $\rightarrow$ AT mutations through the deamination of cytosine and the oxidative conversion of guanine to 8-oxo-guanine (Martin, 1995; Asada, 2006; Murphy, 2009; Shokolenko et al.,
2009). Together, these points suggest that organelle DNAs inhabit a highly mutagenic environment, where DNA repair is inefficient, and the mutational spectrum is skewed toward AT. As one might expect, many species, including most metazoans, appear to have high organelle DNA mutation rates (Lynch et al., 2006). There are some species, however, for which the organelle DNA mutation rate is estimated to be low (e.g., most angiosperms), yet their organelle genomes are still AT-rich (Drouin et al., 2008).

In addition to a genome-wide AT bias, mtDNAs and ptDNAs can exhibit regional and strand-specific nucleotide biases (Gibson, 2005; Kusumi and Tachida, 2005). The mutational consequences of organelle genome replication can give rise to AC vs. GT inequities because the DNA strand that spends more time in the mutationally vulnerable single-stranded state is prone to $\mathrm{C} \rightarrow \mathrm{T}$ and $\mathrm{A} \rightarrow \mathrm{G}$ transitions (Ames et al., 1995; Frank and Lobry, 1999; Faith and Pollock, 2003); but this does not impact the overall AT composition as the G's and T's of one strand are complemented by A's and C's on the other strand.

Natural selection is thought to have contributed to the high AT content of mitochondrial and plastid genomes. Selection for translational efficiency and accuracy is believed to have shaped the nucleotide composition of codons in organelle genes, in some cases enriching the thymine content of synonymous sites (Morton, 1998). Others have argued that AT richness is an adaptation for metabolic efficiency, noting the increased energetic costs of 
producing $\mathrm{C}$ vs. $\mathrm{T}$ and $\mathrm{G}$ vs. $\mathrm{A}$ and the varying abundance of $\mathrm{A} / \mathrm{T}$ vs. G/C nucleotides during organelle DNA synthesis (Jukes and Bhushan, 1986; Wolfe, 1991; Rocha and Danchin, 2002).

Thus, a multitude of forces have likely helped generate the nearuniversal AT bias of organelle DNAs. The discovery of organelle genomes with a high GC content has provided an important point of comparison from which to better understand these forces.

\section{TAXA WITH GC-RICH ORGANELLE GENOMES}

There are more than 40 complete organelle genome sequences in GenBank with GC contents exceeding 50\% (Figure 1). These genomes come from various fish, green algae, and land plants as well as a fungus (Figure 1; Table 1). Moreover, the nucleotide composition of organelle genes, like those encoding the mitochondrial protein cytochrome $\mathrm{c}$ oxidase subunit I $(\operatorname{cox} 1)$ and the large subunit of the plastid protein Rubisco $(r b c L)$, have proven to be good predictors of overall organelle DNA nucleotide content (Min and Hickey, 2007; Clare et al., 2008; Smith, 2009). Analyses of cox 1 and $r b c L$ have revealed other lineages with GC-rich organelle genomes (Kerr et al., 2007; Borza et al., 2009; Figure 2; Table 1). The taxonomic groups containing (or predicted to contain) species with GC-rich organelle DNA are listed below and highlighted in Figures 1-3 and Table 1.

\section{GREEN ALGAE}

Some of the highest organelle genome GC contents come from green algae. The chlorophycean Polytomella capuana, a nonphotosynthetic unicell closely related to the model organism Chlamydomonas reinhardtii, has an mtDNA GC content of $57 \%$ (Smith and Lee, 2008). All other investigated Polytomella species, however, have AT-rich mtDNAs (Smith et al., 2010). Partial mitochondrial genome sequences suggest that the freshwater biflagellates Oogamochlamys gigantea, Lobochlamys segnis, and Lobochlamys culleus, which are also close relatives of C. reinhardtii, have mtDNA GC compositions of approximately 50, 55, and $60 \%$, respectively (Borza et al., 2009). The polar trebouxiophyte Coccomyxa subellipsoidea C-169 has a GC-bias in both its mitochondrial and plastid compartments (53 and 51\% GC, respectively), and organelle gene sequencing indicate that $\mathrm{Coc}$ comyxa chodatii and Coccomyxa rayssiae have GC-rich organelle DNAs as well (Smith et al., 2011). The picoplankton Picocystis salinarum, a deep-branching prasinophyte, appears to have an mtDNA GC content exceeding $60 \%$. And $r b c L$ sequences imply that there are GC-enriched plastid genomes in select members of the charophyte genera Mesotaenium and Hormidiella (Gontcharov and Melkonian, 2010; Rindi et al., 2011).

\section{LAND PLANTS}

The highest recorded GC content in an mtDNA (68\%) and a ptDNA (55\%) belong to the seedless vascular plants Selaginella moellendorffii and Selaginella uncinata, respectively (Tsuji et al., 2007; Hecht et al., 2011). Like C. subellipsoidea, Selaginella species have a GC-bias in both their mitochondrial and plastid compartments (Smith, 2009). Plastid gene sequences of more than 100 Selaginella species from diverse regions revealed only one species without GC-biased ptDNA: the Chinese specimen Selaginella sinensis (Smith, 2009). Other lycophytes, including several Isoetes species, also have relatively high mtDNA GC contents (Malek and Knoop, 1998; Grewe et al., 2009). Analyses of rbcL genes suggests that some ferns from the genera Cheiropleuria, Coniogramme, Cystopteris, Dryopteris, and Monachosorum have a GC-bias in their ptDNA (Ebihara et al., 2010; de Groot et al., 2011).

\section{ANIMALS AND FUNGI}

There are at least 25 species of fish with overall mtDNA GC contents $>50 \%$, such as the wolf herring Chirocentrus dorab (53\%), the Pacific porthole fish Diplophos taenia (52\%), and the beaked salmon Gonorynchus greyi (52\%; Miya and Nishida, 2000; Saitoh et al., 2003; Ishiguro et al., 2005). Moreover, cox1 nucleotide content analyses suggest that potentially hundreds, if not thousands, of other teleosts, from many different orders, have GC-biased mtDNA. Single-gene nucleotide content analyses have revealed various birds, frogs, and corals with GC-rich mitochondrial genomes (Kerr et al., 2007; Crawford et al., 2010). The European penduline tit Remiz pendulinus, the turtle frog Myobatrachus gouldii, and the zoanthid coral Mesozoanthus fossii all appear to have particularly high mtDNA GC contents. Among fungi, the pathogenic yeast Candida subhashii is the only species known to have GC-rich mtDNA (53\%; Fricova et al., 2010).

\section{DIPLONEMIDS}

One of the earliest discoveries of GC-rich mtDNA came from the cox 1 sequence of the euglenozoan Diplonema papillatum, a unicellular phagotrophic marine flagellate (Maslov et al., 1999). Further sequencing of mtDNA from this species has confirmed that its mitochondrial genome is enriched in $\mathrm{G}$ and $\mathrm{C}(\sim 55 \%$; Vlcek et al., 2011). Other investigated members of the genus have AT-rich mtDNAs (Kiethega et al., 2011).

\section{ORGANELLE GENOME ARCHITECTURE AND GC CONTENT}

The available GC-rich organelle genomes (Figures 1 and 2) vary greatly in size, gene content, and coding density (Table 1). For instance, the mtDNA of $P$. capuana is small and compact $(13 \mathrm{~kb}$, $>80 \%$ coding, and no introns; Smith and Lee, 2008) whereas that of S. moellendorffii is large and distended $(250 \mathrm{~kb},>80 \%$ non-coding, and 37 introns; Hecht et al., 2011). There are, however, several reoccurring architectural themes among GC-biased organelle genomes (Table 1).

In the mtDNAs of the chlorophyceans L. culleus and P. capuana and the yeast C. subhashii, a high GC content is partnered with a linear genome conformation and, for the latter two species, distinct telomeric structures (Smith and Lee, 2008; Borza et al., 2009; Fricova et al., 2010). GC-rich mtDNAs are sometimes fragmented into multiple chromosomes, as seen in Oogamochlamys algae and the euglenozoan D. papillatum (Borza et al., 2009; Vlcek et al., 2011); these same taxa, along with P. capuana and S. moellendorffii, also contain fragmented and/or trans-spliced mtDNA genes (Kiethega et al., 2011).

For some species, a high organelle GC content is associated with a small number of tRNA-coding regions: $P$. capuana and $S$. moellendorffii have the most reduced mitochondrial tRNA-coding suites observed from the Archaeplastida: 1 and no tRNAs, respectively. A low tRNA content is also found in the Selaginella plastid genome (Tsuji et al., 2007; Smith, 2009) and the mtDNAs of D. 


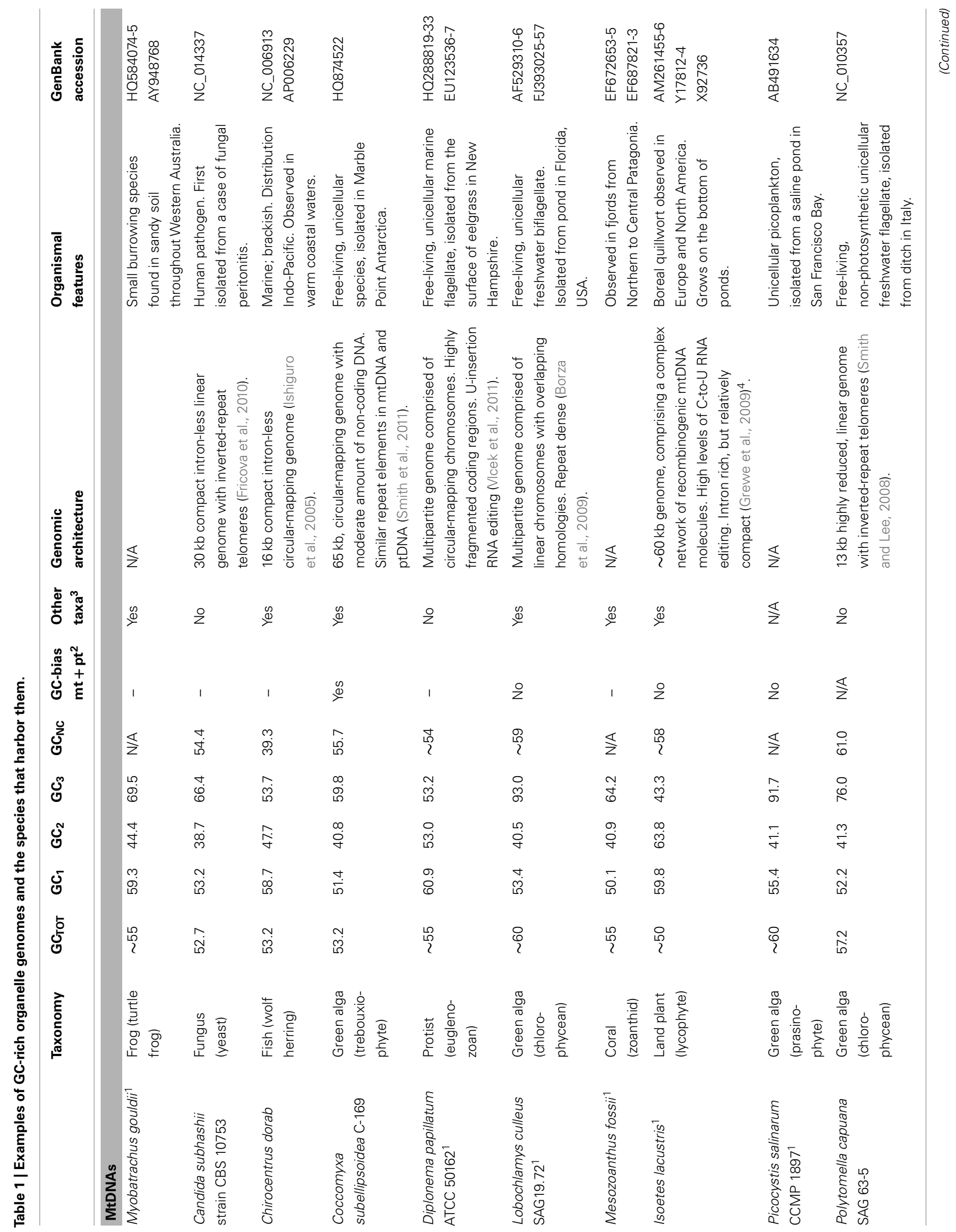




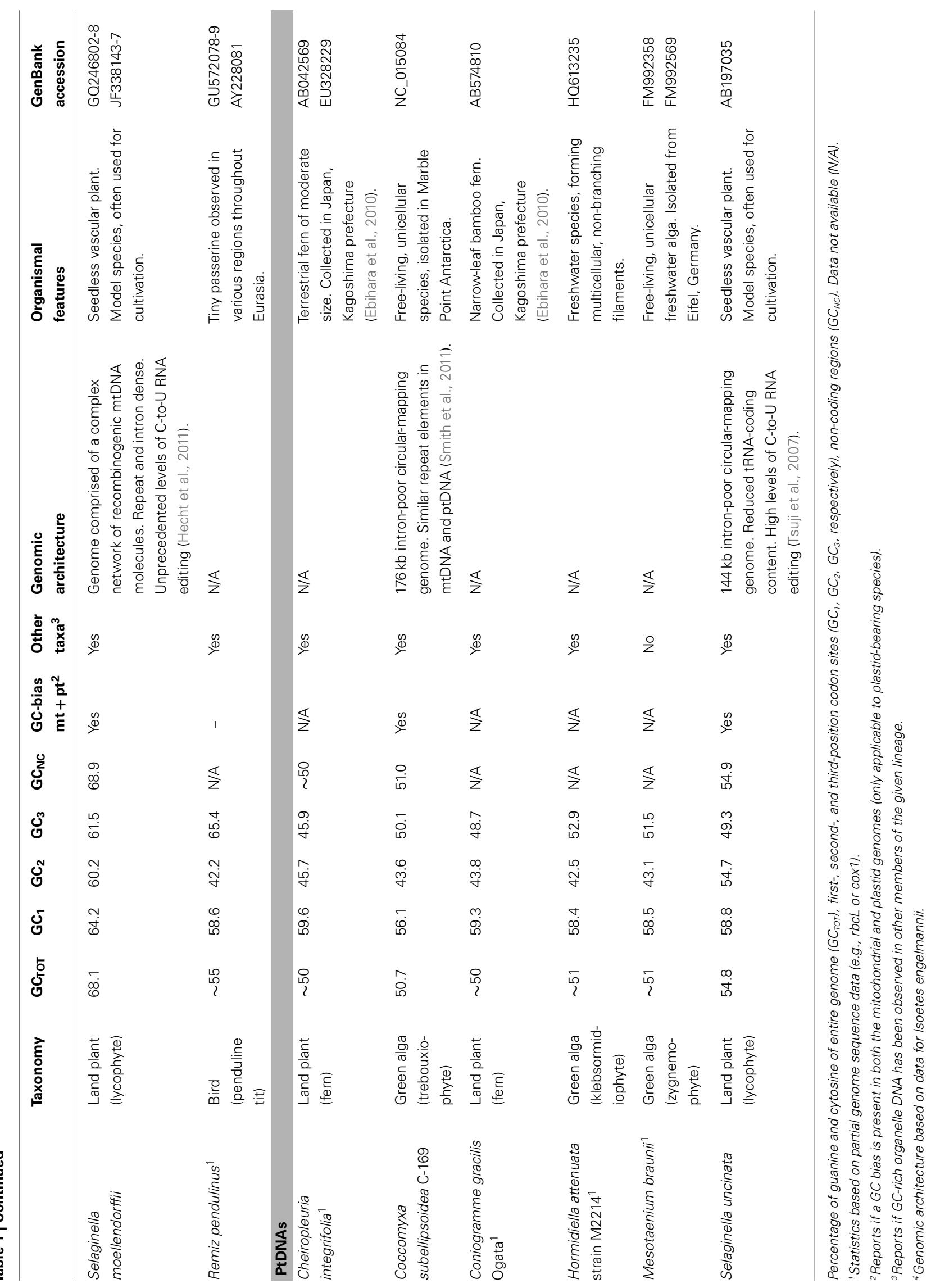




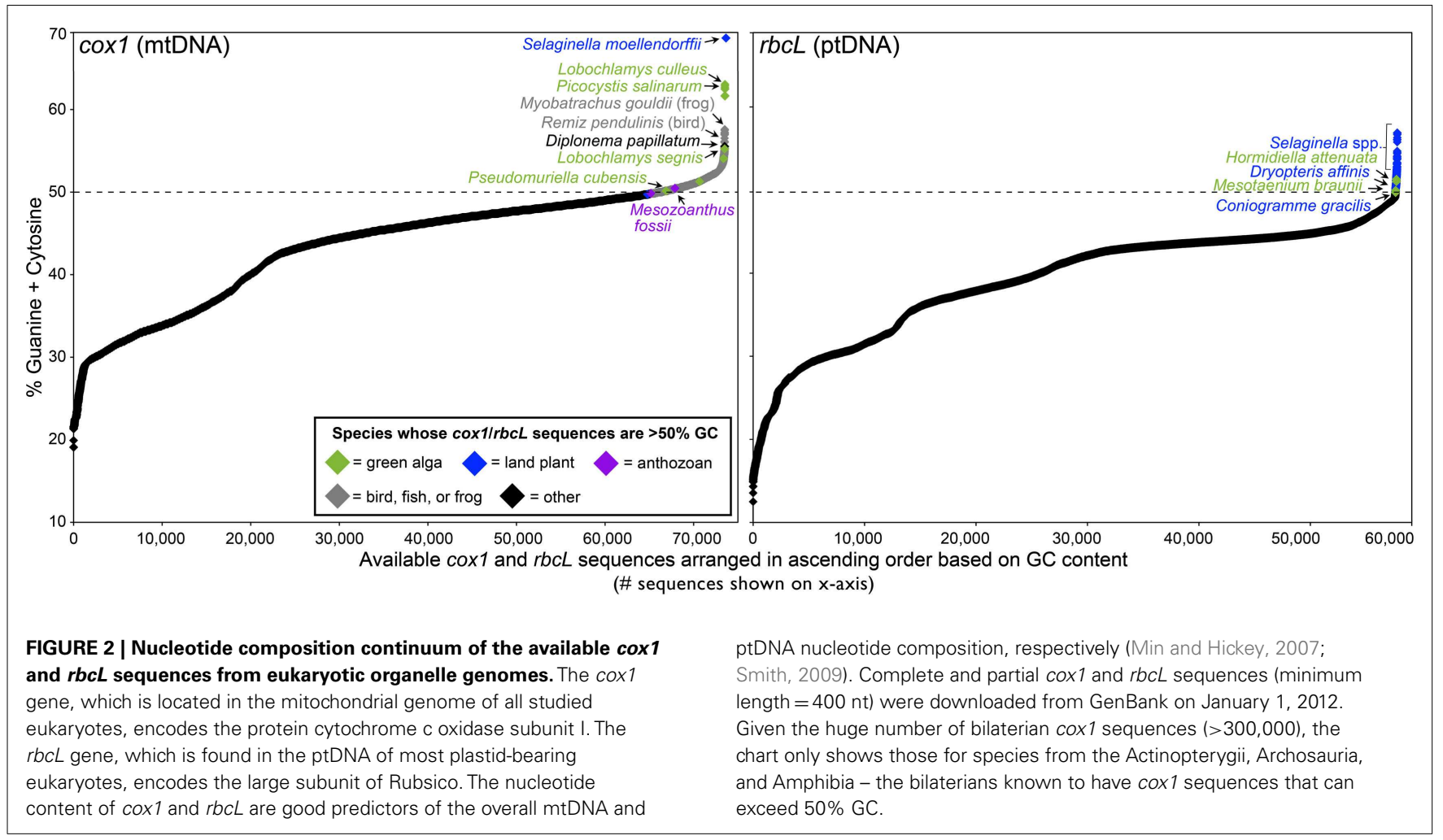

papillatum and zoanthid corals (Sinniger et al., 2007; Vlcek et al., 2011).

In certain cases, organelle genome GC richness is allied with high levels of post-transcriptional editing, particularly cytosineto-uracil changes. Hundreds of $\mathrm{C}$-to- $\mathrm{U}$ editing sites have been identified in the GC-biased mitochondrial and plastid genomes of Selaginella species (Tsuji et al., 2007; Smith, 2009; Hecht et al., 2011). And for land plants as a whole there is a positive relationship between organelle GC content and the abundance of C-to-U editing sites (Jobson and Qiu, 2008). In the GCrich mtDNA of $D$. papillatum, some mitochondrial transcripts experience U-insertion-type RNA editing (Kiethega et al., 2011). Given that organelle RNA editing tends to be a uracil-enriching process, it may turn out that some GC-rich mtDNAs and ptDNAs, once all of their edited sites are uncovered, have AT-rich transcriptomes.

\section{WHAT'S CAUSING ORGANELLE GENOME GC ENRICHMENT?}

Examining the distribution of GC among different regions within a genome, different genomes within a cell, and different species within a group can give insights into the forces that govern GC composition. The available GC-rich organelle DNAs come from an assortment of taxa belonging to disparate lineages (Figure 3). In some cases, the GC-bias is found in both the mitochondrial and plastid compartments of a species and in multiple species within a group, as observed for the spikemoss Selaginella and the trebouxiophyte Coccomyxa (Figure 3). In other examples, the GCbias is restricted to either the mtDNA or ptDNA and/or is present in only a single species within the group, as seen for the green algae Polytomella and Picocystis (Figure 3). This variation in the presence and absence of GC-rich organelle DNA indicates that the processes biasing mitochondrial and plastid genomes in GC likely differ between lineages.

For many GC-rich organelle genomes, particularly those of green algae and the coral Mesozoanthus fossii (Figures 1 and 2), the concentration of GC is highest at silent sites, such as noncoding and synonymous sites (Table 1). This implies that in some organelle systems there is a non-adaptive underpinning to the GC-bias (Kimura, 1983). Two non-adaptive processes that can influence nucleotide landscape are biased mutation pressure and biased gene conversion. In most species, mtDNA and ptDNA mutation pressure seems to be skewed toward $\mathrm{A}$ and $\mathrm{T}$ (discussed above). Gene conversion, however, favors $\mathrm{G}$ and $\mathrm{C}$ in most genomes in which it has been studied (Mancera et al., 2008; Duret and Galtier, 2009; Muyle et al., 2011), with the exception of the tobacco ptDNA where it is AT biased (Khakhlova and Bock, 2006). Genomic regions with high rates of recombination undergo more gene conversion events than those with low recombination rates. In this context, it is noteworthy that some GC-rich organelle genomes are highly recombinogenic (Dieckmann and Gandy, 1987; Smith and Lee, 2008; Borza et al., 2009; Hecht et al., 2011), which may be a sign of a GC-biased conversion process. Moreover, in a variety of organelle genomes, including AT-rich ones, repeat elements (sequences that presumably undergo high levels of recombination) often have inflated GC contents (de Zamaroczy and Bernardi, 1986; Nedelcu and Lee, 1998). DNA methylation can also influence GC content - by promoting cytosine deamination events - but GC-rich mtDNAs and ptDNAs, like those of S. moellendorffii, do not have lower levels of methylation than those that are AT-rich (Zemach et al., 2010). 


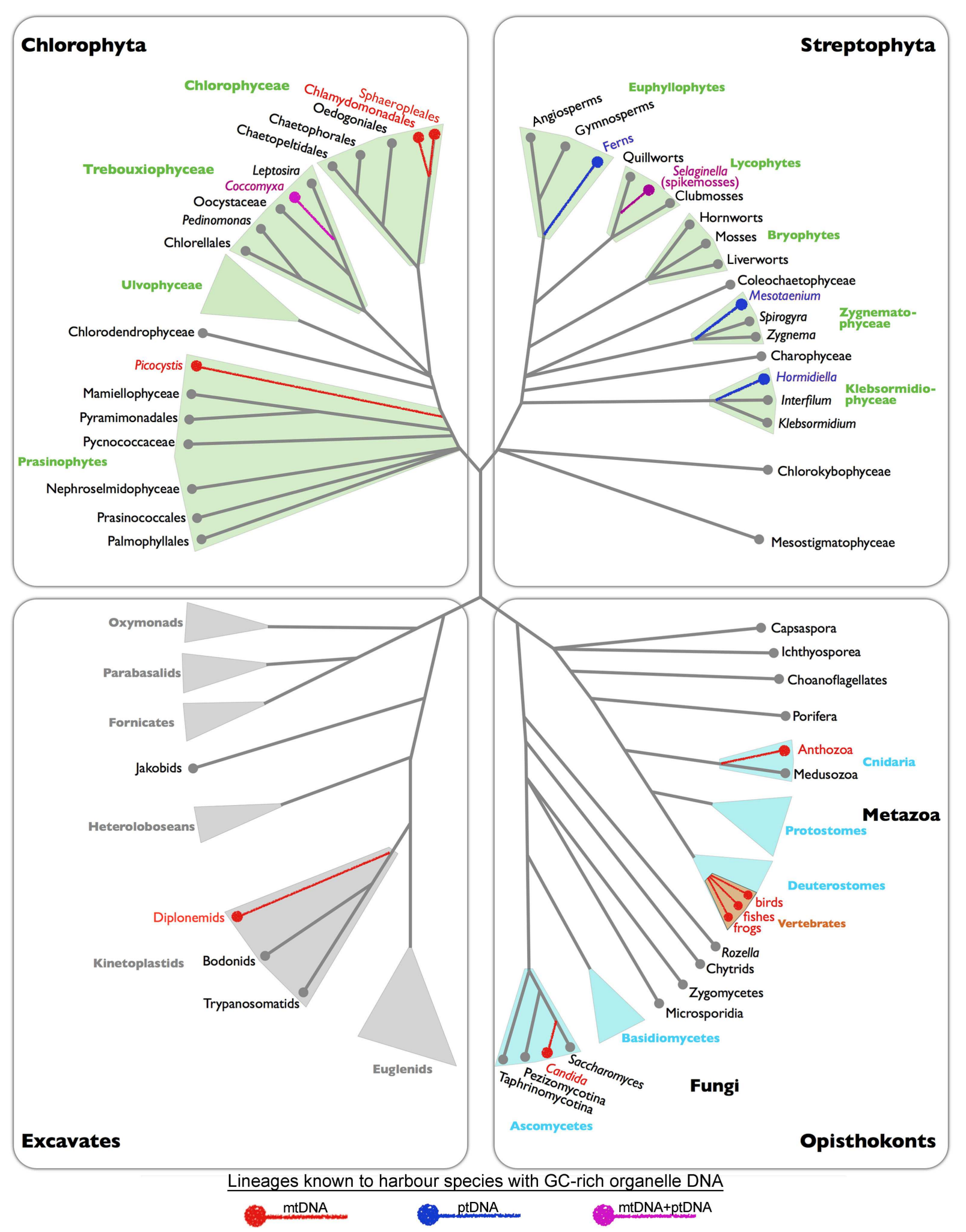

FIGURE 3 | Tree showing the eukaryotic lineages that have species with GC-rich organelle genomes. Colored branches represent lineages that are known to contain (based on complete organelle genome sequence data) or predicted to contain (based on $\operatorname{cox} 1 / \mathrm{rbcL}$ sequences) species with GC-rich organelle genomes (red $=\mathrm{mtDNA}$, blue $=\mathrm{ptDNA}$, pink $=\mathrm{mtDNA}$ and $\mathrm{ptDNA}$ ). Branching order based on published phylogenetic analyses. 
In other organelle DNAs, like those from land plants, the GC content is highest at functionally constrained sites, such as first and second codon positions (Table 1), suggesting that the GC-bias is the product of natural selection. Complicating this interpretation, however, is the fact that many of the cytosines residues at the non-silent sites from these taxa are post-transcriptionally edited to uracil (Jobson and Qiu, 2008; Smith, 2009; Hecht et al., 2011). Other adaptive hypotheses for a high GC composition include increased DNA thermo stability and UV tolerance. But these arguments seem implausible given that many GC-rich organelle DNAs come from species living in extremely cold habitats (e.g., C. subellipsoidea originates from Marble Point Antarctica) or environments with little UV light (e.g., the pathogenic yeast C. subhashii; Table 1).

For some species there is a correlation between lifestyle and organelle DNA GC content. Within the Coccomyxa genus, the three taxa known to have GC-rich organelle genomes are nonlichenized, free-living species, whereas all investigated symbiont Coccomyxa species have AT-rich organelle DNA (Smith et al., 2011). In the case of Candida, a parasitic lifestyle correlates with extreme organelle genome nucleotide compositions: the mtDNA of C. subhashii has one of the highest GC contents observed from the opisthokonts (Fricova et al., 2010) and that of its close relative Candida castellii is remarkably AT-rich (87\%; Figure 1; Bouchier et al., 2009). The high mitochondrial GC contents of certain animals, such as frogs and fish (Figures 1 and 2), may be a reflection of them having low metabolic rates and consequently reduced mtDNA damage from oxygen free radicals (Martin, 1995).

Although data are limited, organelle DNA GC enrichment does not appear to be associated with nuclear DNA GC enrichment: C. subellipsoidea and S. moellendorffii have had their nuclear genomes completely sequenced (Banks et al., 2011; Blanc et al., 2012), revealing overall GC contents of $\sim 50 \%$, which is unremarkable relative to the nuclear genomes of other green plants.

\section{REFERENCES}

Ames, B. N., Shigenaga, M. K., and Hagen, T. M. (1995). Mitochondrial decay in aging. Biochim. Biophys. Acta 1271, 165-170.

Archibald, J. M. (2009). The puzzle of plastid evolution. Curr. Biol. 19, R81-R88.

Asada, K. (2006). Production and scavenging of reactive oxygen species in chloroplasts and their functions. Plant Physiol. 141, 391-396.

Banks, J. A., Nishiyama, T., Hasebe, M., Bowman, J. L., Gribskov, M., dePamphilis, C., Albert, V. A., Aono, N., Aoyama, T., Ambrose, B. A., Ashton, N. W., Axtell, M. J., Barker, E., Barker, M. S., Bennetzen, J. L., Bonawitz, N. D., Chapple, C., Cheng, C., Correa, L. G., Dacre, M., DeBarry, J., Dreyer, I., Elias, M., Engstrom, E. M., Estelle, M., Feng, L., Finet, C., Floyd, S. K., Frommer, W. B., Fujita, T., Gramzow, L., Gutensohn, M., Harholt, J., Hattori, M., Heyl, A., Hirai, T., Hiwatashi, Y., Ishikawa, M.,

However, the availability of these nuclear sequences will allow researchers to explore the full complement of nuclear-encoded mitochondrial- and plastid-targeted proteins, which should give insight into the biochemical and metabolic processes occurring within these organelles. Already, it has been revealed that the C. subellipsoidea nuclear genome lacks the plastid-targeted gene for the photosystem 1 (PSI) reaction center subunit $\mathrm{N}$ (psaN), which codes for a protein involved in the docking of plastocyanin. Interestingly, $p s a N$-lacking strains of Arabidopsis, although maintaining a functional PSI complex, have reduced rates of electron transfer from plastocyanin to PSI (Haldrup et al., 1999). It is hypothesized that for C. subellipsoidea the unique loss of psaN may lead to reduced ROS formation (Blanc et al., 2012), which could help explain the high GC content of its organelle DNAs.

\section{CONCLUDING REMARKS}

Organelle genomes are models for studying the evolution of genome size and structure (Nosek and Tomáska, 2003; Lynch et al., 2006). Now, with the discovery of GC-rich mtDNAs and ptDNAs, they have established themselves as excellent systems for exploring the origins of nucleotide landscape. The presence of GC-biased organelle DNA in key research lineages, like Selaginella, Candida, and Chlamydomonadalean algae, and the availability of complete organelle and nuclear genome sequences from these groups provide promising avenues for future studies on nucleotide composition. I predict that in the years to come GC-rich organelle DNAs will help further our understanding of nucleotide composition and its relationship with other aspects of genome architecture.

\section{ACKNOWLEDGMENTS}

David Roy Smith is supported by postdoctoral fellowships from the Natural Sciences and Engineering Research Council of Canada and the Izaak Walton Killam Trusts.

with the evolution of vascular plants. Science 332, 960-963.

Bendich, A. J. (2010). Mitochondrial DNA, chloroplast DNA and the origins of development in eukaryotic organisms. Biol. Direct 5, 42.

Birky, C. W. (2001). The inheritance of genes in mitochondria and chloroplasts: laws, mechanisms, and models. Annu. Rev. Genet. 35, 125-148.

Blanc, G., Agarkova, I., Grimwood, J., Kuo, A., Brueggeman, A., Dunigan, D. D., Gurnon, J., Ladunga, I., Lindquist, E., Lucas, S., Pangilinan, J., Pröschold, T., Salamov, A., Schmutz, J., Weeks, D., Yamada, T., Lomsadze, A., Borodovsky, M., Claverie, J. M., Grigoriev, I. V., and Van Etten, J. L. (2012). The genome of the polar eukaryotic microalga Coccomyxa subellipsoidea reveals traits of cold adaptation. Genome Biol. 13, R39.

Borza, T., Redmond, E. K., Laflamme, M., and Lee, R. W. (2009).
Mitochondrial DNA in the Oogamochlamys clade (Chlorophyceae): high GC content and unique genome architecture for green algae. J. Phycol. 45, 1323-1334.

Bouchier, C., Ma, L., Créno, S., Dujon, B., and Fairhead, C. (2009). Complete mitochondrial genome sequences of three Nakaseomyces species reveal invasion by palindromic GC clusters and considerable size expansion. FEMS Yeast Res. 9, 1283-1292.

Charlesworth, B., and Charlesworth, D. (2010). Elements of Evolutionary Genetics. Greenwood Village: Roberts and Company Publishers.

Clare, E. L., Kerr, K. C. R., Königslöw, T. E., Wilson, J. J., and Hebert, P. D. N. (2008). Diagnosing mitochondrial DNA diversity: applications of a sentinel gene approach. J. Mol. Evol. $66,362-367$.

Covello, P. S., and Gray, M. W. (1989). RNA editing in plant mitochondria. Nature 341, 662-666. 
Crawford, A. J., Lips, K. R., and Bermingham, E. (2010). Epidemic disease decimates amphibian abundance, species diversity, and evolutionary history in the highlands of central Panama. Proc. Natl. Acad. Sci. U.S.A. 107, 13777-13782.

de Groot, G. A., During, H. J., Maas, J. W., Schneider, H., Vogel, J. C., and Erkens, R. H. J. (2011). Use of rbcL and trnL-F as a two-locus DNA barcode for identification of NW-European ferns: an ecological perspective. PLoS ONE 6, el6371. doi:10.1371/journal.pone.0016371

de Zamaroczy, M., and Bernardi, G. (1986). The GC clusters of the mitochondrial genome of yeast and their evolutionary origin. Gene 41, 1-22.

Dieckmann, C. L., and Gandy, B. (1987). Preferential recombination between GC clusters in yeast mitochondrial DNA. EMBO J. 6, 4197-4203.

Drouin, G., Daoud, H., and Xia, J. (2008). Relative rates of synonymous substitutions in the mitochondrial, chloroplast and nuclear genomes of seed plants. Mol. Phylogenet. Evol. 49, 827-831.

Duret, L., and Galtier, N. (2009). Biased gene conversion and the evolution of mammalian genomic landscapes. Annu. Rev. Genomics Hum. Genet. $10,285-311$.

Ebihara, A., Nitta, J. H., and Ito, M. (2010). Molecular species identification with rich floristic sampling: DNA barcoding the pteridophyte flora of Japan. PLoS ONE 5, e15136. doi:10.1371/journal.pone.0015136

Faith, J. J., and Pollock, D. D. (2003). Likelihood analysis of asymmetrical mutation bias gradients in vertebrate mitochondrial genomes. Genetics 165, 735-745.

Foster, P. G., and Hickey, D. A. (1999). Compositional bias may affect both DNA-based and protein-based phylogenetic reconstructions. J. Mol. Evol. 48, 284-290.

Frank, A. C., and Lobry, J. R. (1999). Asymmetric substitution patterns: a review of possible underlying mutational or selective mechanisms. Gene 238, 65-77.

Fricova, D., Valach, M., Farkas, Z., Pfeiffer, I., Kucsera, J., Tomaska, L., and Nosek, J. (2010). The mitochondrial genome of the pathogenic yeast $\mathrm{Can}$ dida subhashii: GC-rich linear DNA with a protein covalently attached to the $5^{\prime}$ termini. Microbiology 156, 2153-2163.

Gibson, A. (2005). A Comprehensive analysis of mammalian mitochondrial genome base composition and improved phylogenetic methods. Mol. Biol. Evol. 22, 251-264.
Gontcharov, A. A., and Melkonian, M. (2010). Molecular phylogeny and revision of the genus Netrium (Zygnematophyceae, Streptophyta): Nucleotaenium gen. nov. J. Phycol. 46, 346-362.

Gray, M. W., Burger, G., and Lang, B. F. (1999). Mitochondrial evolution. Science 283, 1476-1481.

Gray, M. W., Lang, B. F., and Burger, G. (2004). Mitochondria of protists. Annu. Rev. Genet. 38, 477-524.

Green, B. R. (2011). Chloroplast genomes of photosynthetic eukaryotes. Plant J. 66, 34-44.

Grewe, F., Viehoever, P., Weisshaar, B. and Knoop, V. (2009). A transsplicing group I intron and tRNAhyperediting in the mitochondrial genome of the lycophyte Isoetes engelmannii. Nucleic Acids Res. 37, 5093-5104.

Haldrup, A., Naver, H., and Scheller, H. V. (1999). The interaction between plastocyanin and photosystem I is inefficient in transgenic Arabidopsis plants lacking the PSI-N subunit of photosystem. Plant J. 17, 689-698.

Handa, H. (2008). Linear plasmids in plant mitochondria: peaceful coexistences or malicious invasions. Mitochondrion 8, 15-25.

Hecht, J., Grewe, F., and Knoop, V. (2011). Extreme RNA editing in coding islands and abundant microsatellites in repeat sequences of Selaginella moellendorffii mitochondria: the root of frequent plant mtDNA recombination in early tracheophytes. Genome Biol. Evol. 3, 344-358.

Ishiguro, N. B., Miya, M., Inoue, J. G., and Nishida, M. (2005). Sundasalanx (Sundasalangidae) is a progenetic clupeiform, not a closelyrelated group of salangids (Osmeriformes): mitogenomic evidence. $J$. Fish Biol. 67, 561-569.

Jobson, R. W., and Qiu, Y. L. (2008). Did RNA editing in plant organellar genomes originate under natural selection or through genetic drift? Biol. Direct 3, 43.

Jukes, T. H., and Bhushan, V. (1986). Silent nucleotide substitutions and $\mathrm{G}+\mathrm{C}$ content of some mitochondrial and bacterial genes. J. Mol. Evol. $24,39-44$.

Jukes, T. H., and Osawa, S. (1993). Evolutionary changes in the genetic code. Comp. Biochem. Physiol. B 106, 489-494.

Keeling, P. J. (2010). The endosymbiotic origin, diversification and fate of plastids. Philos. Trans. R. Soc. Lond. B Biol. Sci. 365, 729-748.

Kerr, K. C. R., Stoeckle, M. Y., Dove, C. J., Weigt, L. A., Francis, C. M., and
Hebert, P. D. N. (2007). Comprehensive DNA barcode coverage of North American birds. Mol. Ecol. Notes 7 , 535-543.

Khakhlova, O., and Bock, R. (2006). Elimination of deleterious mutations in plastid genomes by gene conversion. Plant J. 46, 85-94.

Kiethega, G. N., Turcotte, M., and Burger, G. (2011). Evolutionarily conserved coxl trans-splicing without cis-motifs. Mol. Biol. Evol. 28 2425-2428.

Kimura, M. (1983). The Neutral Theory of Molecular Evolution. Cambridge: Cambridge University Press.

Kleine, T., Maier, U. G., and Leister, D. (2009). DNA transfer from organelles to the nucleus: the idiosyncratic genetics of endosymbiosis. Annu. Rev. Plant Biol. 60 115-138.

Kusumi, J., and Tachida, H. (2005). Compositional properties of greenplant plastid genomes. J. Mol. Evol. $60,417-425$.

Lang, B. F., Gray, M. W., and Burger, G. (1999). Mitochondrial genome evolution and the origin of eukaryotes. Annu. Rev. Genet. 33 351-397.

Liu, P., and Demple, B. (2010). DNA repair in mitochondria: much more than we thought? Environ. Mol Mutagen. 51, 417-426.

Lynch, M. (2007). The Origins of Genome Architecture. Massachusetts: Sinauer Associates, Inc.

Lynch, M., Koskella, B., and Schaack, S. (2006). Mutation pressure and the evolution of organelle genomic architecture. Science 311, 1727-1730.

Malek, O., and Knoop, V. (1998). Transsplicing group II introns in plant mitochondria: the complete set of cis-arranged homologs in ferns, fern allies, and a hornwort. RNA 4, 1599-1609.

Mancera, E., Bourgon, R., Brozzi, A., Huber, W., and Steinmetz, L. M. (2008). High-resolution mapping of meiotic crossovers and noncrossovers in yeast. Nature 454 479-485.

Martin, A. P. (1995). Metabolic rate and directional nucleotide substitution in animal mitochondrial DNA. Mol. Biol. Evol. 12, 1124-1131.

Maslov, D. A., Yasuhira, S., and Simpson, L. (1999). Phylogenetic affinities of Diplonema within the Euglenozoa as inferred from the SSU rRNA gene and partial COI protein sequences. Protist 150, 33-42.

McCutcheon, J. P., and Moran, N. A. (2010). Functional convergence in reduced genomes of bacterial symbionts spanning 200 My of evolution. Genome Biol. Evol. 2, 708-718.

Min, X. J., and Hickey, D. A. (2007). DNA barcodes provide a quick preview of mitochondrial genome composition. PLoS ONE 2, e325. doi:10.1371/journal.pone.0000325

Miya, M., and Nishida, M. (2000). Use of mitogenomic information in teleostean molecular phylogenetics: a tree-based exploration under the maximum-parsimony optimality criterion. Mol. Phylogenet. Evol. 17, 437-455.

Moore, C. E., and Archibald, J. M. (2009). Nucleomorph genomes. Annu. Rev. Genet. 43, 251-264.

Morton, B. R. (1998). Selection on the codon bias of chloroplast and cyanelle genes in different plant and algal lineages. J. Mol. Evol. 46, $449-459$.

Muller, H. J. (1964). The relation of recombination to mutational advance. Mutat. Res. 106, 2-9.

Murphy, M. P. (2009). How mitochondria produce reactive oxygen species. Biochem. J. 417, 1-13.

Muyle, A., Serres-Giardi, L., Ressayre, A., Escobar, J., and Glémin, S. (2011). GC-biased gene conversion and selection affect GC content in the Oryza genus (rice). Mol. Biol. Evol. 28, 2695-2706.

Nedelcu, A. M., and Lee, R. W. (1998). Short repetitive sequences in green algal mitochondrial genomes: potential roles in mitochondrial genome evolution. Mol. Biol. Evol. 15, 690-701.

Nosek, J., and Tomáska, L. (2003). Mitochondrial genome diversity: evolution of the molecular architecture and replication strategy. Curr. Genet. 44, 73-84.

Nowack, E. C. M., Melkonian, M., and Glöckner, G. (2008). Chromatophore genome sequence of Paulinella sheds light on acquisition of photosynthesis by eukaryotes. Curr. Biol. 18, 410-418.

Pallen, M. J., and Wren, B. W. (2007). Bacterial pathogenomics. Nature 449, 835-842.

Palmer, J. D. (1985). Comparative organization of chloroplast genomes. Annu. Rev. Genet. 19, 325-354.

Palmer, J. D. (2003). The symbiotic birth and spread of plastids: how many times and whodunit? J. Phycol. 39, $1-9$.

Piganeau, G., Gardner, M., and EyreWalker, A. (2004). A broad survey of recombination in animal mitochondria. Mol. Biol. Evol. 21, 2319-2325. 
Rand, D. M. (2001). The units of selection on mitochondrial DNA. Annu. Rev. Ecol. Syst. 32, 415-448.

Rindi, F., Mikhailyuk, T. I., Sluiman, H. J., Friedl, T., and López-Bautista, J. M. (2011). Phylogenetic relationships in Interfilum and Klebsormidium (Klebsormidiophyceae, Streptophyta). Mol. Phylogenet. Evol. 58, 218-231.

Rocha, E. P., and Danchin, A. (2002). Base composition bias might result from competition for metabolic resources. Trends Genet. 34, 377-378.

Saitoh, K., Miya, M., Inoue, J. G., Ishiguro, N. B., and Nishida, M. (2003). Mitochondrial genomics of ostariophysan fishes: perspectives on phylogeny and biogeography. J. Mol. Evol. 56, 464-472.

Shokolenko, I., Venediktova, N., Bochkareva, A., Wilson, G. L., and Alexeyev, M. F. (2009). Oxidative stress induces degradation of mitochondrial DNA. Nucleic Acids Res. 37, 2539-2548.

Simpson, L., and Thiemann, O. H. (1995). Sense from nonsense: RNA editing in mitochondria of kinetoplastid protozoa and slime molds. Cell 81, 837-840.

Sinniger, F., Chevaldonné, P., and Pawlowski, J. (2007). Mitochondrial genome of Savalia savaglia (Cnidaria, Hexacorallia) and early metazoan phylogeny. J. Mol. Evol. 64, 196-203.

Smith, D. R. (2009). Unparalleled GC content in the plastid DNA of Selaginella. Plant Mol. Biol. 71, 627-639.

Smith, D. R., Burki, F., Yamada, T., Grimwood, J., Grigoriev, I. V., Van Etten, J. L., and Keeling, P. J. (2011). The GC-rich mitochondrial and plastid genomes of the green alga Coccomyxa give insight into the evolution of organelle DNA nucleotide landscape. PLoS ONE 6, e23624. doi:10.1371/journal.pone.0023624

Smith, D. R., Hua, J., and Lee, R. W. (2010). Evolution of linear mitochondrial DNA in three known lineages of Polytomella. Curr. Genet. 56, 427-438.

Smith, D. R., and Lee, R. W. (2008). Mitochondrial genome of the colorless green alga Polytomella capuana: a linear molecule with an unprecedented GC content. Mol. Biol. Evol. 25, 487-496.

Tsuji, S., Ueda, K., Nishiyama, T., Hasebe, M., Yoshikawa, S., Konagaya, A., Nishiuchi, T., and Yamaguchi, K. (2007). The chloroplast genome from a lycophyte (microphyllophyte), Selaginella uncinata, has a unique inversion, transpositions and many gene losses. J. Plant Res. 120, 281-290.

Vlcek, C., Marande, W., Teijeiro, S., Lukes, J., and Burger, G. (2011). Systematically fragmented genes in a multipartite mitochondrial genome. Nucleic Acids Res. 39, 979-988.

Wolfe, K. H. (1991). Mammalian DNA replication: mutation biases and the mutation rate. J. Theor. Biol. 149, 441-451.

Wu, M., Zhang, L., Li, G., Jiang, D. and Ghabrial, S. A. (2010). Genome characterization of a debilitationassociated mitovirus infecting the phytopathogenic fungus Botrytis cinerea. Virology 406, 117-126.

Zemach, A., McDaniel, I. E., Silva, P., and Zilberman, D. (2010). Genome-wide evolutionary analysis of eukaryotic
DNA methylation. Science 328, 916-919.

Conflict of Interest Statement: The author declares that the research was conducted in the absence of any commercial or financial relationships that could be construed as a potential conflict of interest.

Received: 30 May 2012; paper pending published: 19 July 2012; accepted: 20 August 2012; published online: 11 September 2012.

Citation: Smith DR (2012) Updating our view of organelle genome nucleotide landscape. Front. Gene. 3:175. doi: 10.3389/fgene.2012.00175

This article was submitted to Frontiers in Plant Genetics and Genomics, a specialty of Frontiers in Genetics.

Copyright (C) 2012 Smith. This is an open-access article distributed under the terms of the Creative Commons Attribution License, which permits use, distribution and reproduction in other forums, provided the original authors and source are credited and subject to any copyright notices concerning any third-party graphics etc. 